\title{
Prenatal and Adolescent Exposure to Tobacco Smoke Modulates the Development of White Matter Microstructure
}

\author{
Leslie K. Jacobsen, ${ }^{1,2,9}$ Marina R. Picciotto, ${ }^{1,3,4}$ Christopher J. Heath, ${ }^{8}$ Stephen J. Frost, ${ }^{9}$ Kristen A. Tsou, ${ }^{1}$ Rita A. Dwan, \\ Marcel P. Jackowski, ${ }^{10}$ Robert T. Constable, ${ }^{5,6,7}$ and W. Einar Mencl ${ }^{9}$ \\ Departments of ${ }^{1}$ Psychiatry, ${ }^{2}$ Pediatrics, ${ }^{3}$ Neurobiology, ${ }^{4}$ Pharmacology, ${ }^{5}$ Diagnostic Radiology, ${ }^{6}$ Neurosurgery, and ${ }^{7}$ Biomedical Engineering, and \\ ${ }^{8}$ Interdepartmental Neuroscience Program, Yale University School of Medicine, New Haven, Connecticut 06519, ${ }^{9}$ Haskins Laboratories, New Haven, \\ Connecticut 06511, and ${ }^{10}$ Institute of Mathematics and Statistics, University of São Paulo, CEP 05508-090, São Paulo, Brazil
}

Prenatal exposure to maternal smoking has been linked to cognitive and auditory processing deficits in offspring. Preclinical studies have demonstrated that exposure to nicotine disrupts neurodevelopment during gestation and adolescence, possibly by disrupting the trophic effects of acetylcholine. Given recent clinical and preclinical work suggesting that neurocircuits that support auditory processing may be particularly vulnerable to developmental disruption by nicotine, we examined white matter microstructure in 67 adolescent smokers and nonsmokers with and without prenatal exposure to maternal smoking. The groups did not differ in age, educational attainment, IQ, years of parent education, or symptoms of inattention. Diffusion tensor anisotropy and anatomical magnetic resonance images were acquired, and auditory attention was assessed, in all subjects. Both prenatal exposure and adolescent exposure to tobacco smoke was associated with increased fractional anisotropy (FA) in anterior cortical white matter. Adolescent smoking was also associated with increased FA of regions of the internal capsule that contain auditory thalamocortical and corticofugal fibers. FA of the posterior limb of the left internal capsule was positively correlated with reaction time during performance of an auditory attention task in smokers but not in nonsmokers. Development of anterior cortical and internal capsule fibers may be particularly vulnerable to disruption in cholinergic signaling induced by nicotine in tobacco smoke. Nicotine-induced disruption of the development of auditory corticofugal fibers may interfere with the ability of these fibers to modulate ascending auditory signals, leading to greater noise and reduced efficiency of neurocircuitry that supports auditory processing.

Key words: adolescent; prenatal; tobacco; white matter microstructure; thalamocortical fibers; corticofugal fibers

\section{Introduction}

Clinical studies have linked maternal smoking during pregnancy with deficits in auditory processing and general intellectual function, and with attention deficit hyperactivity disorder in offspring (McCartney et al., 1994; Romano et al., 2006). Nicotine binds to nicotinic acetylcholine receptors (nAChRs), which, when stimulated by endogenous acetylcholine, regulate brain development (Slotkin, 2004). Stimulation of nAChRs by nicotine during gestation disrupts neurodevelopment, possibly by disrupting the trophic actions of acetylcholine (Navarro et al., 1989; Slotkin, 2004). Nicotine administration during the early postnatal period in the rodent disrupts auditory learning and nicotinic regulation of primary auditory cortex (Hsieh et al., 2002; Liang et al., 2006). High-affinity nAChRs expressed on corticothalamic efferents

\footnotetext{
Received May 25, 2007; revised Sept. 21, 2007; accepted 0ct. 24, 2007.

This work was supported by U.S. Public Health Service Grants DA14655, DA017333, and M01 RR000125; M.R.P. and C.J.H. were supported by DA00436, AA15632, and MH77681. Biolmage Suite was developed with support from National Institutes of Health Grant R01 EB006494. We thank Dr. Jonathan S. Feinstein for statistical consultation, and the subjects and their families for their participation.

Correspondence should be addressed to Leslie K. Jacobsen, Departments of Psychiatry and Pediatrics, Yale University School of Medicine, 2 Church Street South, Suite 207, New Haven, CT 06519. E-mail: leslie.jacobsen@yale.edu.

DOI:10.1523/JNEUROSCI.2402-07.2007

Copyright $\odot 2007$ Society for Neuroscience $\quad$ 0270-6474/07/2713491-08\$15.00/0
}

during development are critical for normal passive avoidance learning (King et al., 2003). Stimulation of nAChRs by nicotine may also alter timing of the switch in action of GABA from excitation to inhibition, which may disrupt circuit development (Liu et al., 2006).

Nicotine is also disruptive to adolescent brain development (Abreu-Villaca et al., 2003). Animals exposed to nicotine during both prenatal and adolescent development, demonstrate effects on cholinergic neurotransmission, cell signaling, and serotonin receptor expression that are more prominent than those observed after exposure during either prenatal or adolescent development alone, particularly in females (Slotkin et al., 2007). Parallel work in humans demonstrated that female adolescents with combined prenatal and adolescent exposure to tobacco smoke had greater impairments of both auditory and visual attention than females with exposure during either prenatal or adolescent development alone, whereas male adolescents with combined exposure had prominent impairments in auditory attention (Jacobsen et al., 2007). Exposure to tobacco smoke during prenatal and/or adolescent development was associated with reduced efficiency of neurocircuits activated during auditory attention, including primary auditory cortex (Jacobsen et al., 2007).

Auditory signals travel from the cochlea through brainstem, midbrain, and the medial geniculate body (MGB) of the thala- 
Table 1. Demographic, clinical, and cognitive characteristics of 33 adolescents with and 34 adolescents without prenatal exposure to active maternal smoking

\begin{tabular}{|c|c|c|c|c|}
\hline Characteristic & $\begin{array}{l}\text { Smokers, prenatally } \\
\text { exposed }(N=25)\end{array}$ & $\begin{array}{l}\text { Smokers, no } \\
\text { prenatal exposure } \\
(N=14)\end{array}$ & $\begin{array}{l}\text { Nonsmokers, } \\
\text { prenatally exposed } \\
(N=8)\end{array}$ & $\begin{array}{l}\text { Nonsmokers, no } \\
\text { prenatal exposure } \\
(N=20)\end{array}$ \\
\hline Age (years) & $16.3 \pm 1.1$ & $17.0 \pm 0.7$ & $16.2 \pm 1.4$ & $16.3 \pm 1.2$ \\
\hline Gender (M/F) & $7 / 18$ & $7 / 7$ & $4 / 4$ & $8 / 12$ \\
\hline Education (years) & $9.2 \pm 1.4$ & $9.9 \pm 1.1$ & $9.4 \pm 1.5$ & $9.6 \pm 1.2$ \\
\hline Parent education (years) & $15.5 \pm 5.3$ & $14.1 \pm 2.8$ & $13.1 \pm 1.9$ & $15.0 \pm 2.2$ \\
\hline Cigarettes smoked/day & $11.6 \pm 6.5$ & $9.8 \pm 6.9$ & & \\
\hline Age at onset of smoking & $12.5 \pm 2.2$ & $13.1 \pm 1.8$ & & \\
\hline Years of daily smoking & $2.6 \pm 1.7$ & $3.1 \pm 1.8$ & & \\
\hline Estimated plasma nicotine (7:30 P.M.) & $13.1 \pm 8.7$ & $12.5 \pm 7.3$ & & \\
\hline FTND $^{a}$ score $^{b}$ & $4.6 \pm 1.8$ & $3.1 \pm 1.8$ & & \\
\hline Weeks prenatal exposure to tobacco & $32.4 \pm 11.7$ & 0.0 & $22.3 \pm 16.1$ & 0.0 \\
\hline Weeks prenatal exposure to environmental tobacco smoke ${ }^{c}$ & $21.5 \pm 20.2$ & $19.8 \pm 20.6$ & $30.1 \pm 18.6$ & $12.4 \pm 18.2$ \\
\hline Birth weight (kg) & $3.22 \pm 0.40$ & $3.35 \pm 0.48$ & $3.28 \pm 0.42$ & $3.35 \pm 0.52$ \\
\hline $\mathrm{KBIT}^{d}$ composite score & $97.4 \pm 8.9$ & $99.5 \pm 9.8$ & $98.5 \pm 13.2$ & $104.5 \pm 13.0$ \\
\hline WJR Word Attack SS ${ }^{e}$ & $108.7 \pm 16.3$ & $108.4 \pm 14.7$ & $106.2 \pm 16.0$ & $107.3 \pm 18.0$ \\
\hline Beck Depression Score ${ }^{f, g}$ & $6.5 \pm 6.8$ & $7.2 \pm 6.9$ & $2.9 \pm 3.8$ & $2.3 \pm 3.2$ \\
\hline Conners $^{h}$ Score & $20.7 \pm 13.2$ & $17.8 \pm 7.6$ & $16.4 \pm 11.5$ & $15.0 \pm 7.6$ \\
\hline Rate of alcohol consumption (drinks/week) ${ }^{i}$ & $1.7 \pm 2.4$ & $3.1 \pm 3.7$ & $0.1 \pm 0.2$ & $0.4 \pm 0.8$ \\
\hline Lifetime episodes of cannabis use $^{j}$ & $194.0 \pm 286.8$ & $339.9 \pm 442.5$ & $0.5 \pm 0.8$ & $47.5 \pm 134.5$ \\
\hline
\end{tabular}

Data are presented as mean $\pm S D$, unless otherwise specified.

${ }^{a}$ Fagerstrom Test for Nicotine Dependence (Heatherton et al., 1991).

${ }^{b}$ Prentally exposed $>$ no prenatal exposure: $\beta=1.5, t=2.5, p<0.05$.

Prentally exposed $>$ no prenatal exposure: $\beta=17.7, t=2.2, p<0.05$.

${ }^{d}$ Kaufman Brief Intelligence Test (Bowers and Pantle, 1998).

${ }^{e}$ Woodcock-Johnson Revised Test of Achievement Word Attack subtest standard scores.

fBeck et al., 1961.

${ }^{9}$ Smokers $>$ nonsmokers: $\beta=4.9, t=2.5, p<0.05$.

${ }^{h}$ Conners Adolescent Self Report Scale (Conners, 1998).

iSmokers $>$ nonsmokers: $\beta=2.7, t=3.4, p<0.01$.

'Smokers $>$ nonsmokers: $\beta=337.4, t=3.3, p<0.01$.

mus before reaching primary auditory cortex. Auditory cortex neurons project back to the ipsilateral MGB, thalamic reticular nucleus, and bilateral inferior colliculi (Ehret and Romand, 1997; $\mathrm{He}, 2003)$. These descending fibers form the corticofugal projections and, together with corticothalamic fibers, pass through the internal capsule (Parent, 1996). We tested for evidence of alternations in white matter microstructure associated with prenatal and adolescent exposure to tobacco smoke using diffusion tensor imaging (DTI). DTI provides quantitative measures of the diffusion of water within tissue (Beaulieu, 2002). Directionality [fractional anisotropy (FA)] of water diffusion increases with increasing age in children and adolescents across white matter pathways (Barnea-Goraly et al., 2005b), and correlates with developmental changes in cognitive abilities (Mabbott et al., 2006). Given that nicotine may disrupt the trophic actions of acetylcholine at nAChRs, we hypothesized that prenatal and/or adolescent exposure to tobacco smoke would be associated with increases in FA of the internal capsule and that, among subjects with developmental exposure to tobacco smoke, increased internal capsule FA would be correlated with impairments in auditory attention. Because the internal capsule also includes corticospinal fibers, we examined the relationship between internal capsule FA and motor speed to test the specificity of the effect of tobacco smoke exposure on auditory corticothalamic and corticofugal fibers.

\section{Materials and Methods}

Participants. Thirty-three adolescents with prenatal exposure to active maternal smoking were compared with 34 adolescents with no prenatal exposure to active maternal smoking. Prenatally exposed subjects included 25 current daily tobacco smokers and 8 nonsmokers (defined as having a lifetime history of smoking no more than two cigarettes), whereas subjects with no prenatal exposure to maternal smoking included 14 current daily tobacco smokers and 20 nonsmokers. Participants were 13-18 years of age and were recruited from the community by advertisement. All subjects were free of medical and psychiatric illness and substance abuse or dependence disorders, other than nicotine dependence, as determined by physical exam and structured clinical interview (Sobell and Sobell, 1992; Meyers et al., 1995; Kaufman et al., 1997). Detailed drug use history was obtained using semistructured interviews and the Comprehensive Addiction Severity Index for Adolescents (Meyers et al., 1995). Abstinence from substance use during the week before assessment was confirmed by urine toxicology screen.

At initial screening, general intelligence was estimated using the Kaufman Brief Intelligence Test (Bowers and Pantle, 1998), reading achievement was estimated using the Word Attack subtest of the WoodcockJohnson Revised Test of Achievement, and self-reported symptoms of depression and inattention were assessed using the Beck Depression Scale (Beck et al., 1961) and the Conners Adolescent Self Report Scale (Conners, 1998), respectively. Prenatal exposure was assessed by semistructured interview of the parents regarding the average number of cigarettes smoked per day by the mother during each trimester of her pregnancy with the subject. The mother's consumption of other substances and alcohol during the pregnancy, and the rate of smoking by other persons living in the home during the pregnancy (prenatal environmental exposure), were also assessed during this interview. Demographic, clinical, and cognitive characteristics of both groups are presented in Table 1.

The groups did not differ in age, gender, symptoms of inattention, years of education, years of parent education, or birth weight. Smokers reported significantly more symptoms of depression at screening $(\beta=$ $4.9 ; t=2.5 ; p<0.05)$, and greater history of alcohol $(\beta=2.7 ; t=3.4 ; p<$ $0.01)$ and cannabis $(\beta=337.4 ; t=3.3 ; p<0.01)$ consumption. These effects of adolescent smoking were not significantly modified by prenatal exposure to maternal smoking. Adolescents with prenatal exposure to 
maternal smoking had more environmental tobacco smoke exposure during gestation (persons other than the mother smoking in the home during gestation of the subject; $\beta=17.7 ; t=2.2 ; p<0.05$ ). This effect of prenatal exposure was not significantly modified by smoking status. Consistent with previous evidence that maternal smoking during pregnancy increases risk of smoking in offspring (Kandel et al., 1994; Griesler et al., 1998; Cornelius et al., 2000; Buka et al., 2003), smokers with prenatal exposure to maternal smoking reported more symptoms of nicotine dependence, as measured by the Fagerstrom Test for Nicotine Dependence (Heatherton et al., 1991) $(\beta=1.5 ; t=2.5 ; p<0.05)$. Effects of prenatal exposure to maternal smoking on age at onset of smoking, years of daily smoking, and number of cigarettes smoked per day were not significant.

Among the adolescents with prenatal exposure to maternal smoking, exposure was restricted to the first trimester in 7, whereas 6 were exposed through two trimesters, and 20 were exposed throughout gestation. Reported maternal use of other drugs during pregnancy was as follows: among mothers of smokers with prenatal exposure to maternal smoking, six consumed alcohol, two consumed cannabis, and two consumed cocaine during the index pregnancy. Among mothers of smokers without prenatal exposure to maternal smoking, two consumed alcohol, none consumed cannabis, and one consumed cocaine during the pregnancy. Among mothers of nonsmokers with prenatal exposure to maternal smoking, two consumed alcohol, one consumed cannabis, and one consumed cocaine during the pregnancy. Among mothers of nonsmokers without prenatal exposure to maternal smoking, none consumed alcohol, cannabis, or cocaine during the pregnancy. No subject in the sample was exposed to opiates, amphetamine, hallucinogens, sedativehypnotics, or inhalants during gestation. Adolescents with prenatal exposure to active maternal smoking also had more prenatal exposure to alcohol $(\beta=0.4 ; t=2.5 ; p<0.05)$. This effect was not significantly modified by smoking status. Rates of prenatal exposure to cannabis or cocaine did not significantly differ across the groups.

Seven smokers with prenatal exposure to maternal smoking had experimented with illicit substances other than cannabis; four had tried cocaine, one had tried amphetamine, four had tried oral opiates, two had tried sedative-hypnotics, and four had tried hallucinogens. Five smokers with no prenatal exposure to maternal smoking had experimented with illicit substances other than cannabis; four had tried cocaine, one had tried amphetamine, two had tried oral opiates, one had tried sedativehypnotics, and one had tried hallucinogens. Nonsmokers with and without prenatal exposure to maternal smoking denied previous use of cocaine, amphetamine, opiates, sedative hypnotics, and hallucinogens, and all subjects denied previous use of methylenedioxymethamphetamine (ecstasy), inhalants, anabolic steroids, and injected drugs.

Parental consent was obtained for subjects 17 years of age and younger. This study was approved by the Yale University School of Medicine Human Investigation Committee (Yale HIC 12230). Subjects provided written assent or, for 18 year olds, consent for study participation.

Assessment of attention and motor speed. As previously described (Jacobsen et al., 2007), subjects performed a modified version of a computerized word recognition task involving progressively more demanding manipulations of attention (Shaywitz et al., 2001; Shafritz et al., 2004). Trials within each task were $5 \mathrm{~s}$ long and began with a $500 \mathrm{~ms}$ visual cue, depicting an eye or an ear, prompting subjects to attend to the visual or auditory modality, respectively. After a $500 \mathrm{~ms}$ pause, a word or nonword was presented in the cued modality. Subjects then made a word/nonword discrimination (lexical decision) with a button press. In the simple condition, word or nonword stimuli were presented only in the attended modality, whereas nonlinguistic stimuli (four diagonal lines or a tone stimulus) were presented in the unattended modality. In the selective attention condition, a linguistic stimulus (word or nonword) was simultaneously presented in the unattended modality, thereby placing greater demand on neurocircuits mediating perceptual selectivity.

Motor speed was assessed using a computerized version of the Finger Tapping Test (Western Psychological Services, Los Angeles, CA), in which average tapping rate for each hand was computed from five $10 \mathrm{~s}$ trials. In addition, all subjects completed the Grooved Pegboard Test (Lafayette Instruments, Lafayette, IN), in which 25 ridged pegs are placed in a $5 \times 5$ matrix of grooved holes in a prescribed order. The score for each hand was the time required to complete the task (Ruff and Parker, 1993; Schmidt et al., 2000).

Image acquisition. Subjects were scanned using a 3.0 tesla Siemens (Erlangen, Germany) Trio magnetic resonance imaging (MRI) system and received a three-dimensional, high-resolution T1-weighted anatomic scan using a sagittal magnetization-prepared rapid acquisition with gradient-echo (MPRAGE) pulse sequence: echo time (TE), $3.66 \mathrm{~ms}$; repetition time (TR), $2530 \mathrm{~ms}$; flip angle, $7^{\circ}$; field of view (FOV), $256 \times$ 256 pixels; slice thickness, $1 \mathrm{~mm}$; 176 slices; 0 skip. A standard quadrature head coil was used for both radio frequency transmission and magnetic resonance signal reception. Axial DTI images were acquired using a single shot echoplanar sequence (TE, $84 \mathrm{~ms}$; TR, $7500 \mathrm{~ms}$; FOV, $320 \mathrm{~mm}$; matrix, $128 \times 128$; slice thickness, $2.5 \mathrm{~mm}$; 56 slices; 0 skip; $b=1000$ $\mathrm{s} / \mathrm{mm}^{2}$ ). Diffusion was measured along six noncollinear directions. For each of these six gradient directions, two acquisitions were averaged. Two acquisitions without diffusion weighting $\left(b=0 \mathrm{~s} / \mathrm{mm}^{2}\right)$ were also averaged. Thus, seven DTI volumes were obtained in total.

Data analysis. Image processing was performed using locally developed software written in Matlab (MathWorks, Natick, MA). Voxelwise FA maps were calculated from the diffusion tensor within BioImage Suite (www.bioimagesuite.org; New Haven, CT) (Basser and Pierpaoli, 1996). Before across-subjects comparisons, DTI data were spatially normalized to standard stereotactic space as follows. First, the high-resolution anatomic (MPRAGE) scan was stripped of skull tissue using the brain extraction tool (BET) (Smith, 2002) within FSL (www.fmrib.ox.ac.uk/fsl), and the rigid alignment of the subject's T2-weighted $b=0 \mathrm{~s} / \mathrm{mm}^{2}$ image to his/her stripped high resolution anatomic image was determined. Then, the subject's high-resolution anatomic scan was normalized to standard stereotactic space (Montreal Neurologic Institute Colin Template: http://www.bic.mni.mcgill.ca/brainweb) using nonlinear spatial normalization (Collins et al., 1998; Holmes et al., 1998; Ashburner and Friston, 1999; Papademetris et al., 2003). Before analysis, images were smoothed using a $4 \mathrm{~mm}$ full width at half-maximum (FWHM) Gaussian filter. A $4 \mathrm{~mm}$ FWHM filter was chosen on the basis of previous DTI studies in adolescent populations (Barnea-Goraly et al., 2005a,b). Finally, a white matter mask was created from the spatially normalized MNI Colin brain and applied to the DTI images to restrict analyses to data acquired from white matter.

Effects of prenatal exposure to maternal smoking and adolescent smoking status on FA were assessed at each white matter voxel using mixed-model repeated-measures ANOVA (Woods, 1996), with prenatal exposure and smoking status as between-subjects variables, and subject treated as a random effect within each group. Voxelwise correlation analyses were conducted to examine the relationships between white matter FA and the number of weeks of prenatal exposure to maternal smoking, magnitude of tobacco use during adolescence (pack-years), speed (reaction time) and accuracy (proportion correct) of auditory attention task performance, and measures of motor speed. A univariate voxel threshold of $p<0.01$, corrected for mapwise false discovery rate (FDR) (Genovese et al., 2002), was used, with an additional cluster threshold of 10 contiguous significant voxels. Location of peak group differences in FA were converted to Talairach and Tournoux coordinate space (Talairach and Tournoux, 1988), using the nonlinear transformation by Brett (www. mrc-cbu.cam.ac.uk/Imaging/Common/mnispace.shtml).

\section{Results}

Effects of prenatal exposure to maternal smoking and adolescent smoking on white matter fractional anisotropy Significant group differences in FA observed by voxelwise repeated-measures ANOVA are presented in Figure 1, where results of the comparison of nonsmokers with prenatal exposure to maternal smoking (exposed nonsmokers) to nonsmokers with no prenatal exposure (nonexposed nonsmokers) are presented in the left panel, results of the comparison of smokers with no prenatal exposure (nonexposed smokers) to nonexposed nonsmokers are presented in the middle panel, and results of the comparison of smokers with prenatal expo- 
sure to maternal smoking (exposed smokers) to nonexposed nonsmokers are presented in the right panel. Images are displayed per radiological convention, with the right side of the brain on the left side of the figure. Regions where prenatal and/or adolescent exposure to tobacco smoke was associated with significant increases in FA are shown in red/yellow, whereas regions where exposure was associated with significant decreases in fractional anisotropy are shown in blue/purple. Talairach coordinates of regions showing significant effects of prenatal and/or adolescent exposure to tobacco smoke on FA are provided

Table 2.

Prenatal exposure to maternal smoking alone was associated with increased FA in right and left frontal regions and in the genu of the corpus callosum (Fig. 1, left). Adolescent smoking in the absence of prenatal exposure was associated with increased FA in a larger number of frontal regions, the genu and splenium of the corpus callosum, the left inferior longitudinal fasciculus, and in the anterior limb of the right internal capsule (Fig. 1, middle). Prenatal exposure to maternal smoking combined with adolescent smoking was associated with increased FA in a larger volume of regions relative to the other two exposure groups, including the genu of the corpus callosum, left frontal white matter, the right superior longitudinal fasciculus, and the anterior limb of the right internal capsule. All regions showing increased FA among subjects with prenatal and/or adolescent exposure to tobacco smoke relative to nonexposed nonsmokers remained significant after controlling for potential confounding variables, including baseline symptoms of depression (Beck score), gender, alcohol use, lifetime episodes of cannabis use, prenatal exposure to environmental tobacco smoke, and prenatal exposure to maternal alcohol consumption. Decreased FA was observed in one region of the left external capsule among subjects with combined prenatal and adolescent exposure relative to nonexposed nonsmokers. However, this difference did not remain significant after controlling for potential confounding variables.

Direct comparison of adolescent smokers with and without prenatal exposure revealed no significant differences in regional FA. However, direct comparison of adolescent smokers with no prenatal exposure to prenatally exposed nonsmokers revealed that FA was significantly greater in adolescent smokers with no prenatal exposure in the anterior limb of the right internal capsule (Talairach coordinates: $x=38, y=24, z=12$; volume, 352 $\left.\mathrm{mm}^{3}\right)$.

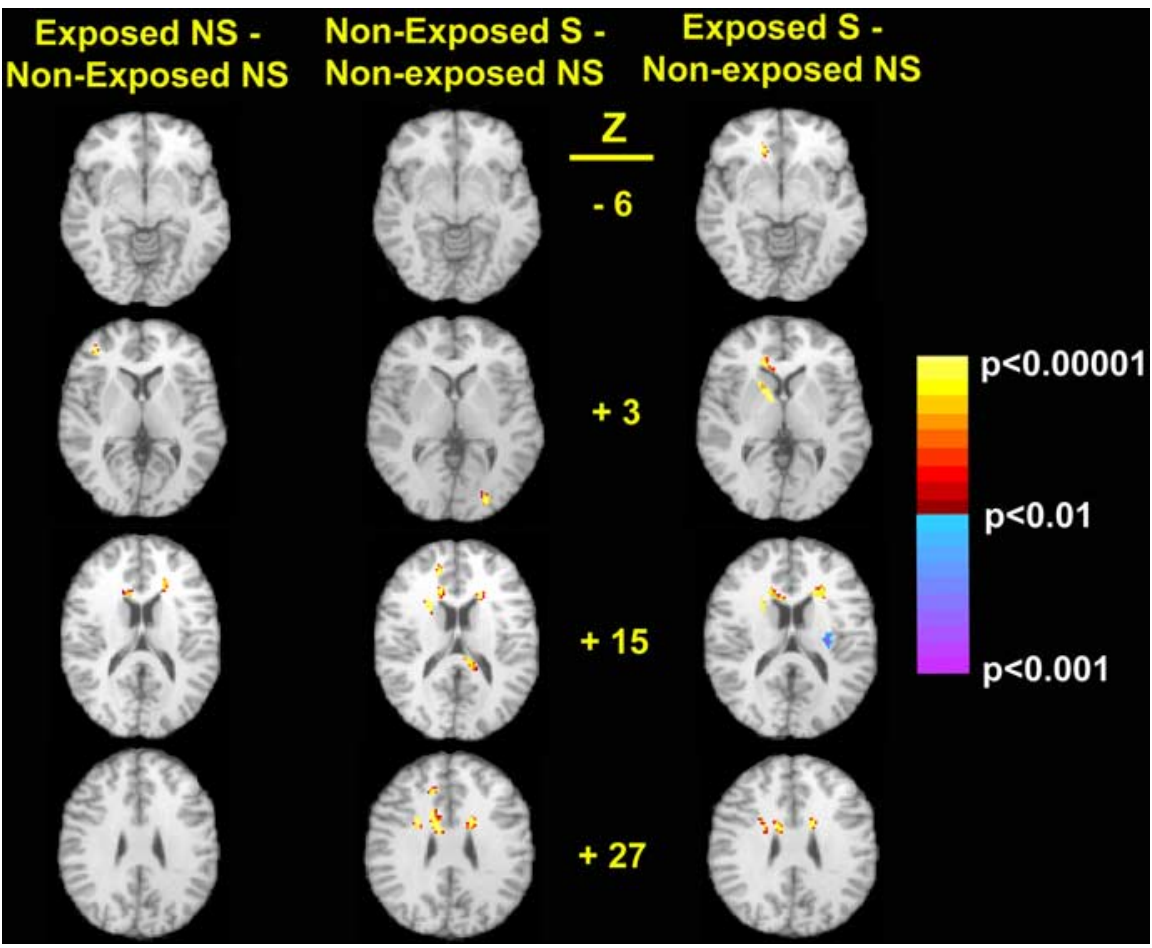

Figure 1. Results of voxelwise repeated-measures ANOVA assessments of the effects of prenatal and adolescent exposure to tobacco smoke on white matter FA. Left panel, Results of the comparison of nonsmokers with prenatal exposure to maternal smoking (Exposed NS) to nonsmokers with no prenatal exposure to maternal smoking (NonExposed NS). Middle panel, Results of the comparison of smokers with no prenatal exposure (NonExposed S) to nonexposed NS. Right panel, Results of the comparison of smokers with prenatal exposure to maternal smoking (Exposed S) to nonexposed NS.

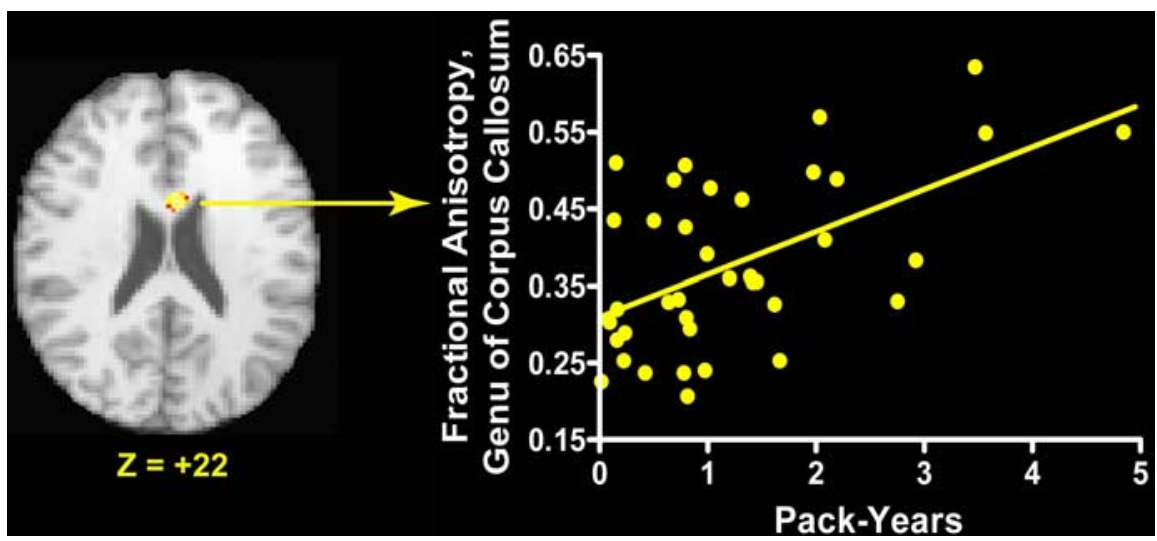

Figure 2. Left, Results of voxelwise correlation analysis examining the relationship between white matter FA and magnitude of tobacco smoke exposure during adolescence (pack-years), showing a significant positive correlation between FA of the genu of the corpus callosum and pack-years among smokers. Right, Plot of FA values from the region of the genu of the corpus callosum showing a significant positive correlation with pack-years.

Relationships between measures of prenatal and adolescent exposure to tobacco smoke, auditory attention, motor speed, and white matter fractional anisotropy

Among subjects with prenatal exposure to maternal smoking, the number of weeks of prenatal exposure was not significantly correlated with white matter FA. Among smokers, pack-years was significantly positively correlated with FA at the genu of the corpus callosum (Talairach coordinates: $x=-4, y=5, z=22$; volume, 408 $\mathrm{mm}^{2}$ ) (Fig. 2). FA data were extracted from the region of the genu showing a significant correlation with pack-years and were submitted to regression analysis. Analysis revealed that the relationship between genu FA and pack-years remained significant after controlling 
Table 2. Regions demonstrating significant effects of prenatal exposure to maternal smoking and adolescent smoking on FA

\begin{tabular}{|c|c|c|c|c|c|}
\hline \multirow[b]{2}{*}{ Structure } & \multicolumn{5}{|c|}{ Talairach coordinates } \\
\hline & $x$ & $y$ & $Z$ & Volume $\left(\mathrm{mm}^{3}\right)$ & Peak $p$ value $^{a}$ \\
\hline \multicolumn{6}{|c|}{ Structures with increased FA in prenatally exposed NS relative to nonexposed NS } \\
\hline R frontal short association fibers & 40 & 45 & 1 & 88 & 0.0002 \\
\hline L frontal white matter & -20 & 32 & 13 & 160 & 0.002 \\
\hline R genu, corpus callosum & 12 & 22 & 14 & 80 & 0.002 \\
\hline \multicolumn{6}{|c|}{ Structures with increased FA in nonexposed S relative to nonexposed NS } \\
\hline L inferior longitudinal fasciculus & -24 & -85 & 4 & 120 & 0.0008 \\
\hline R forceps minor, corpus callosum & 14 & 30 & 12 & 328 & 0.0001 \\
\hline R internal capsule, anterior limb & 24 & 16 & 14 & 456 & $<0.0001$ \\
\hline R frontal association fibers & 16 & 45 & 14 & 192 & $<0.0001$ \\
\hline L splenium, corpus callosum & -8 & -34 & 15 & 384 & 0.001 \\
\hline L forceps minor, corpus callosum & -18 & 18 & 18 & 520 & $<0.0001$ \\
\hline R genu, corpus callosum & 12 & 9 & 24 & 792 & 0.0001 \\
\hline R superior longitudinal fasciculus & 28 & 7 & 26 & 200 & 0.0001 \\
\hline $\mathrm{R}$ frontal short association fibers & 16 & 32 & 24 & 80 & 0.0008 \\
\hline \multicolumn{6}{|c|}{ Structures with increased FA in exposed S relative to nonexposed NS } \\
\hline R internal capsule, anterior limb & 15 & 10 & 4 & 2608 & $<0.0001$ \\
\hline R genu, corpus callosum & 10 & 27 & 4 & 2528 & 0.0001 \\
\hline L frontal white matter & -22 & 28 & 12 & 1544 & $<0.0001$ \\
\hline R genu, corpus callosum & 12 & 3 & 26 & 128 & 0.0009 \\
\hline R superior longitudinal fasciculus & 24 & 3 & 26 & 96 & 0.001 \\
\hline L forceps minor, corpus callosum & -16 & 5 & 26 & 96 & 0.0007 \\
\hline \multicolumn{6}{|c|}{ Structures with decreased FA in exposed S relative to nonexposed NS } \\
\hline L external capsule & -30 & -13 & 12 & 1064 & 0.0003 \\
\hline
\end{tabular}

Voxel threshold: $\alpha=0.01$, FDR corrected; cluster threshold: 10 contiguous significant voxels. R, Right; L, left. $x, y$, and $z$ are Talairach coordinates of the peak difference in FA.

${ }^{a} p$ value of peak group difference in $\mathrm{FA}$.

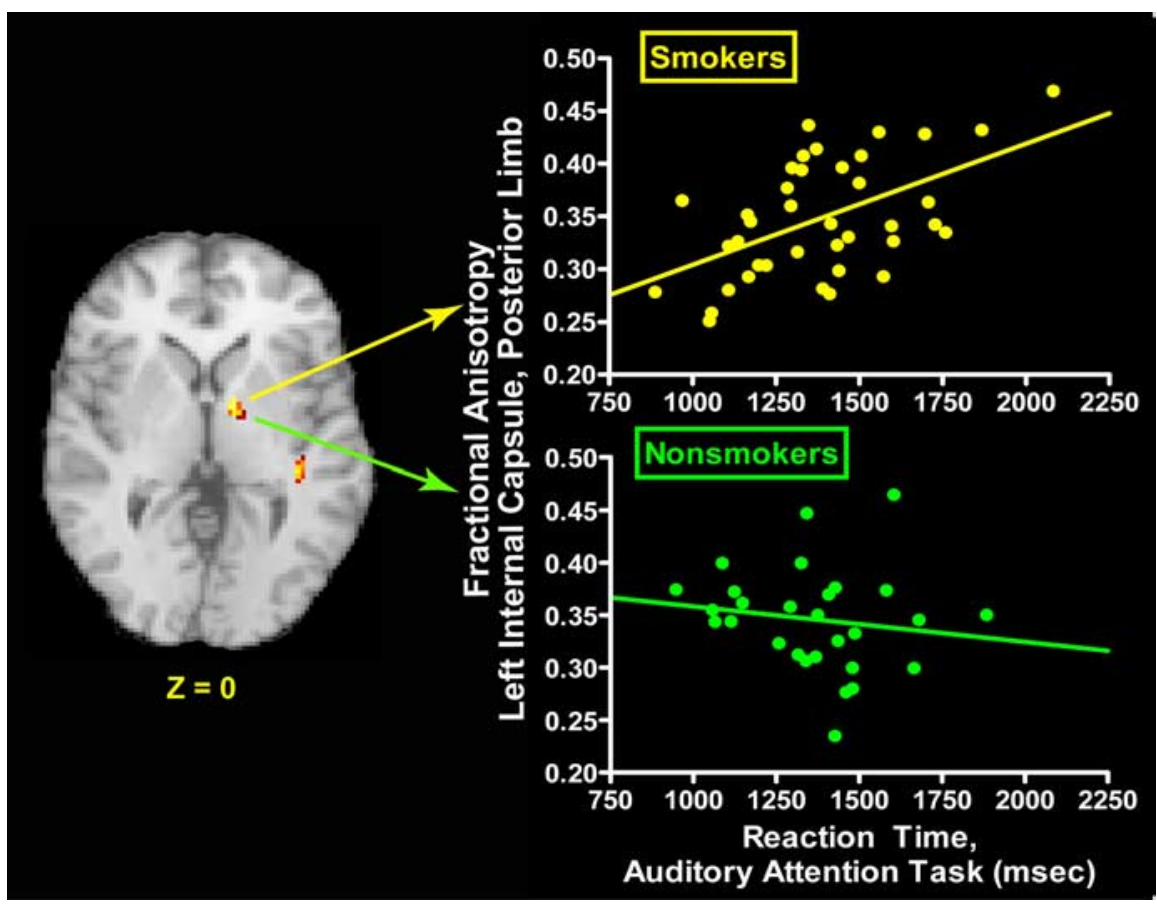

Figure 3. Left, Results of voxelwise correlation analysis examining the relationship between FA of the internal capsule and reaction time during auditory attention task performance among smokers. Right, Plot of auditory attention task reaction time and FA values from the region of the left posterior internal capsule showing a significant positive correlation with auditory attention task reaction time among smokers (top plot) but not among nonsmokers (bottom plot) (adolescent smoking by FA of left posterior internal capsule interaction effect: $\beta=2632.8, t=2.2, p=0.03$ ).

for potential confounding variables and was not significantly modified by prenatal exposure to maternal smoking.

Our a priori hypothesis was that effects of developmental exposure to tobacco smoke on thalamocortical and corticothalamic fibers may contribute to tobacco smoke exposure-related deficits in auditory attention. We tested this hypothesis by examining the relationship between auditory attention task performance (accuracy and reaction time) and FA within the internal capsule, where these fibers are located (Parent, 1996; Mori et al., 2005). Because effects of adolescent exposure to tobacco smoke on white matter FA were prominent, analyses were conducted within smokers and nonsmokers. FA of the internal capsule was not significantly correlated with accuracy of auditory attention task performance for either smokers or nonsmokers. Among smokers, reaction time during the auditory attention task was significantly positively correlated with FA of the posterior limb of the left internal capsule (Talairach coordinates: $x=-10, y=-2, z=0$; volume, $192 \mathrm{~mm}^{2}$ ) (Fig. 3, left), and with FA of the white matter of the left temporal lobe (Talairach coordinates: $x=-42, y=$ $-27, z=0$; volume, $168 \mathrm{~mm}^{2}$ ) (supplemental Fig. 1, available at www.jneurosci.org as supplemental material). The weak inverse relationship between FA of the internal capsule and auditory attention task reaction time among nonsmokers was not significant. Regression analysis of FA data extracted from the region of the left posterior internal capsule showing a significant correlation with auditory attention task reaction time among smokers demonstrated that this relationship remained significant after controlling for potential confounding variables and was not significantly modified by prenatal exposure to maternal smoking (adolescent smoking by FA of left posterior internal capsule interaction effect: $\beta=2632.8, t=2.2, p=$ $0.03)$ (Fig. 3, right). 
Effects of prenatal exposure to maternal smoking and adolescent smoking on tapping rate during the Finger Tapping Test and on time to complete the Grooved Pegboard Test were not significant. Neither Finger Tapping Test nor Grooved Pegboard Test measures of motor speed were significantly correlated with internal capsule FA within the sample as a whole, or within smokers and nonsmokers.

\section{Discussion}

In the present study, exposure to tobacco smoke during prenatal development alone, adolescent development alone, or during both developmental epochs was associated with significant increases in regional white matter FA, primarily in anterior cortical and subcortical regions. In addition to myelination, FA increases reflect maturational increases in cell packing density, fiber diameter, and directional coherence (Beaulieu and Allen, 1994; Shimony et al., 1999; Beaulieu, 2002). Thus, observed group differences are consistent with previous evidence that stimulation of nAChRs by nicotine may disrupt neurodevelopment by disrupting the trophic actions of acetylcholine (Slotkin, 1998; Oncken et al., 2003). FA increases were most extensive in adolescent smokers with and without prenatal exposure. Furthermore, increased right anterior internal capsule FA was observed in adolescent smokers without prenatal exposure relative to prenatally exposed nonsmokers. This pattern suggests that white matter maturation may be somewhat affected by prenatal nicotine exposure, but is particularly vulnerable to the disruptive effects of nicotine during adolescence, perhaps because of the prominent developmental changes in cortical and subcortical white matter that occur during this period (Giedd et al., 1999; Paus et al., 1999; BarneaGoraly et al., 2005b; Ashtari et al., 2007).

The significant positive correlation between the magnitude of tobacco exposure during adolescence and FA of the genu of the corpus callosum, further supports the notion that effects of nicotine on white matter maturation may be particularly significant during adolescence. nAChRs are expressed on oligodendrocyte precursors (Rogers et al., 2001), and thus the effect of adolescent tobacco exposure on white matter FA may reflect a greater effect of nicotine on these precursors during adolescence. Direct comparison of adolescent smokers with and without prenatal exposure to maternal smoking showed no significant differences in FA, suggesting that the effect of adolescent exposure to tobacco smoke on white matter microstructure is not significantly magnified by prenatal exposure to maternal smoking. Thus, although there may be a small contribution of nicotine exposure during prenatal development to changes in FA, effects on this pathway would not be additive with adolescent exposure, perhaps because nicotine acts on similar mechanisms during the prenatal and adolescent period, and thus the effect of prenatal exposure is occluded in individuals with both prenatal and adolescent exposure.

FA of regions of anterior cortical white matter, including the genu of the corpus callosum and fibers that project into the frontal lobes from the corpus callosum, the thalamus, and the superior longitudinal fasciculus, was increased in all three exposure groups. Adolescent tobacco use, both with and without prenatal exposure to maternal smoking, was further associated with increased FA of the anterior limb of the right internal capsule. In addition to corticospinal and corticopontine fibers, the internal capsule contains thalamocortical and corticofugal fibers (Parent, 1996; Mori et al., 2005). Although thalamocortical projections are restricted to the posterior limb of the internal capsule, corticofugal projections, which comprise a larger proportion of inter- nal capsule fibers than thalamocortical projections, are present in both anterior and posterior limbs of the internal capsule (Andersen et al., 1980; Parent, 1996; Winer et al., 2001; Mori et al., 2005). Convergent lines of evidence have shown that corticofugal fibers modulate acoustic information ascending through auditory pathways (Suga et al., 2002; Perrot et al., 2006; Winer, 2006; Sun et al., 2007;), permitting top-down control and optimization of auditory signal processing (Suga et al., 2002; Perrot et al., 2006; Sun et al., 2007). Human postmortem work has shown that thalamic and basal telencephalic fibers extending to the fetal auditory cortex contain cholinergic markers throughout development of central auditory neurocircuitry and are detectable as early as 10.5 weeks of gestation (Krmpotic-Nemanic et al., $1983 a, b)$. Cholinergic markers appear in synaptic layers of auditory cortex at 24-26 weeks of gestation, likely reflecting thalamic innervation of the auditory cortex (Krmpotic-Nemanic et al., 1980). Studies in fetal cat suggest that efferent projections extending from auditory cortex are detectable by midgestation and reach the thalamus during the third trimester (Payne et al., 1988; Payne, 1992). Longitudinal structural MRI and diffusion tensor imaging studies have provided evidence of ongoing maturation of internal capsule fibers during adolescence, reflected by increases in white matter density and FA during this period (Paus et al., 1999; Ashtari et al., 2007).

Work in rodents has demonstrated evidence that nicotine exposure during the neonatal period, which corresponds to the third trimester in humans, disrupts synaptic development in auditory cortex, producing deficits in auditory learning (Aramakis et al., 2000; Liang et al., 2006). In addition, expression of highaffinity $\mathrm{nAChRs}$ in corticothalamic projections during development is necessary for correct performance of an aversive learning task in mice, suggesting that development of this pathway is particularly vulnerable to disruptions in cholinergic signaling (King et al., 2003). We recently reported evidence that, in humans, vulnerability to disrupting effects of nicotine in tobacco smoke on the development of auditory neurocircuitry extends into adolescence, producing deficits in auditory attention and reduced efficiency of neurocircuitry that supports auditory attention (Jacobsen et al., 2007). In the present study, reaction time during performance of an auditory attention task was positively correlated with increasing FA of the posterior limb of the left internal capsule in smokers but weakly inversely related to FA of this region in nonsmokers. Although causality cannot be inferred from these associations, these observations are consistent with our hypothesis that developmental exposure to tobacco smoke disrupts the development of auditory thalamocortical and corticofugal fibers possibly by disrupting the trophic effects of acetylcholine at nAChRs. In particular, nicotine induced maldevelopment of corticofugal fibers may interfere with the ability of these fibers to modulate ascending auditory signals, leading to greater noise and reduced efficiency of the circuit (Suga et al., 2002; He, 2003; Sun et al., 2007). The lack of a significant correlation between finger tapping and grooved pegboard measures of motor speed and internal capsule FA among smokers or nonsmokers further supports the specificity of the effect of tobacco smoke exposure on development of thalamocortical and corticofugal fibers and suggests that corticopontine and corticospinal fibers may be less vulnerable to developmental disruption resulting from tobacco smoke exposure.

The possibility that the group differences in cortical and subcortical white matter FA observed in the present study stem from factors unrelated to prenatal or adolescent exposure to nicotine in tobacco smoke cannot be excluded. However, the fact that 
regional increases in white matter FA associated with prenatal and/or adolescent exposure to tobacco smoke remained significant after group matching and statistical controls for potential confounding variables, including cannabis and alcohol use and maternal use of alcohol, argues against this possibility, as does the congruence of many of our conclusions with the findings from rodent models of exposure to nicotine alone. Sample size did not permit evaluation of the degree to which gender modifies the effect of prenatal and adolescent exposure to tobacco smoke on white matter microstructure. Other limitations include the measurement of prenatal exposure by retrospective self-report. Work comparing prospectively and retrospectively collected data about pregnancy has supported the accuracy of pregnancy data collected by retrospective self-report (Jacobson et al., 1991; Buka et al., 2004). Similarly, comparison of serum cotinine concentrations and self-reported smoking behavior during pregnancy has yielded evidence of significant agreement between these measures (Buka et al., 2003). Finally, the possibility that genetic factors shared by mothers and their offspring may mediate both increased risk for smoking and alterations in white matter microstructure cannot be excluded.

In summary, the present findings suggest that exposure to tobacco smoke during prenatal and adolescent developmental epochs disrupts the development of white matter microstructure, particularly in anterior cortical regions and in the internal capsule. This effect of exposure to tobacco smoke on development of white matter microstructure may result from nicotine in tobacco smoke disrupting the trophic effects of acetylcholine at nAChRs. Effects of tobacco smoke exposure were pronounced when exposure occurred during adolescence, raising the possibility that the extensive maturational changes that occur in white matter during adolescence may confer vulnerability to developmental disruption by nicotine (Giedd et al., 1999; Paus et al., 1999; BarneaGoraly et al., 2005b; Ashtari et al., 2007). FA of the posterior limb of the left internal capsule was positively correlated with reaction time during auditory attention task performance in smokers but was only weakly inversely related to speed of auditory attention in nonsmokers, supporting the notion that nicotine-induced disruption of the development of auditory thalamocortical and corticofugal may reduce the efficiency of this neurocircuit. Together with previous human evidence linking developmental exposure to tobacco smoke with auditory processing deficits and reduced efficiency of neurocircuitry that supports auditory processing (McCartney et al., 1994; Jacobsen et al., 2007), and rodent data demonstrating that developmental exposure to nicotine disrupts the development of synapses in auditory cortex and corticothalamic projections (Aramakis et al., 2000; King et al., 2003; Liang et al., 2006), the present findings suggest that development of auditory thalamocortical and corticofugal pathways is particularly vulnerable to disruptions in cholinergic signaling induced by nicotine in tobacco smoke.

\section{References}

Abreu-Villaca Y, Seidler FJ, Tate CA, Slotkin TA (2003) Nicotine is a neurotoxin in the adolescent brain: critical periods, patterns of exposure, regional selectivity, and dose thresholds for macromolecular alterations. Brain Res 979:114-128.

Andersen RA, Knight PL, Merzenich MM (1980) The thalamocortical and corticothalamic connections of AI, AII, and the anterior auditory field (AAF) in the cat: evidence for two largely segregated systems of connections. J Comp Neurol 194:663-701.

Aramakis VB, Hsieh CY, Leslie FM, Metherate R (2000) A critical period for nicotine-induced disruption of synaptic development in rat auditory cortex. J Neurosci 20:6106-6116.
Ashburner J, Friston KJ (1999) Nonlinear spatial normalization using basis functions. Hum Brain Mapp 7:254-266.

Ashtari M, Cervellione KL, Hasan KM, Wu J, McIlree C, Kester H, Ardekani BA, Roofeh D, Szeszko PR, Kumra S (2007) White matter development during late adolescence in healthy males: a cross-sectional diffusion tensor imaging study. NeuroImage 35:501-510.

Barnea-Goraly N, Eliez S, Menon V, Bammer R, Reiss AL (2005a) Arithmetic ability and parietal alterations: a diffusion tensor imaging study in velocardiofacial syndrome. Brain Res Cogn Brain Res 25:735-740.

Barnea-Goraly N, Menon V, Eckert M, Tamm L, Bammer R, Karchemskiy A, Dant CC, Reiss AL (2005b) White matter development during childhood and adolescence: a cross-sectional diffusion tensor imaging study. Cereb Cortex 15:1848-1854.

Basser PJ, Pierpaoli C (1996) Microstructural and physiological features of tissues elucidated by quantitative-difusion-tensor MRI. J Magn Reson B 111:209-219.

Beaulieu C (2002) The basis of anisotropic water diffusion in the nervous system-a technical review. NMR Biomed 15:435-455.

Beaulieu C, Allen PS (1994) Determinants of anisotropic water diffusion in nerves. Magn Reson Med 31:394-400.

Beck AT, Ward CH, Mendelson M, Mock J, Erbaugh J (1961) An inventory for measuring depression. Arch Gen Psychiatry 4:561-571.

Bowers TL, Pantle ML (1998) Shipley institute for living scale and the Kaufman Brief Intelligence Test as screening instruments for intelligence. Assessment 5:187-195.

Buka SL, Shenassa ED, Niaura R (2003) Elevated risk of tobacco dependence among offspring of mothers who smoked during pregnancy: a 30-year prospective study. Am J Psychiatry 160:1978-1984.

Buka SL, Goldstein JM, Spartos E, Tsuang MT (2004) The retrospective measurement of prenatal and perinatal events: accuracy of maternal recall. Schizophr Res 71:417-426.

Collins DL, Zijdenbos AP, Kollokian V, Sled JG, Kabani NJ, Holmes CJ, Evans AC (1998) Design and construction of a realistic digital brain phantom. IEEE Trans Med Imaging 17:463-468.

Conners CK (1998) Rating scales in attention-deficit/hyperactivity disorder: use in assessment and treatment monitoring. J Clin Psychiatry 59 [Suppl 7]:24-30.

Cornelius MD, Leech SL, Goldschmidt L, Day N (2000) Prenatal tobacco exposure: is it a risk factor for early tobacco experimentation? Nicotine Tob Res 2:45-52.

Ehret G, Romand R (1997) The central auditory system. New York: Oxford UP.

Genovese CR, Lazar NA, Nichols T (2002) Thresholding of statistical maps in functional neuroimaging using the false discovery rate. NeuroImage 15:870-878.

Giedd JN, Blumenthal J, Jeffries NO, Castellanos FX, Liu H, Zijdenbos A, Paus T, Evans AC, Rapoport JL (1999) Brain development during childhood and adolescence: a longitudinal MRI study. Nat Neurosci 2:861-863.

Griesler PC, Kandel DB, Davies M (1998) Maternal smoking in pregnancy, child behavior problems, and adolescent smoking. J Res Adolesc 8:159-185.

He J (2003) Corticofugal modulation of the auditory thalamus. Exp Brain Res 153:579-590.

Heatherton TF, Kozlowski LT, Frecker RC, Fagerstrom KO (1991) The Fagerstrom test for nicotine dependence: a revision of the Fagerstrom Tolerance Questionnaire. Br J Addict 86:1119-1127.

Holmes CJ, Hoge R, Collins L, Woods R, Toga AW, Evans AC (1998) Enhancement of MR images using registration for signal averaging. J Comput Assist Tomogr 22:324-333.

Hsieh CY, Leslie FM, Metherate R (2002) Nicotine exposure during a postnatal critical period alters NR2A and NR2B mRNA expression in rat auditory forebrain. Brain Res Dev Brain Res 133:19-25.

Jacobsen LK, Slotkin TA, Mencl WE, Frost SJ, Pugh KR (2007) Gender specific effects of prenatal and adolescent exposure to tobacco smoke on auditory and visual attention. Neuropsychopharmacology 32:2453-2464.

Jacobson SW, Jacobson JL, Sokol RJ, Martier SS, Ager JW, Kaplan MG (1991) Maternal recall of alcohol, cocaine, and marijuana use during pregnancy. Neurotoxicol Teratol 13:535-540.

Kandel DB, Wu P, Davies P (1994) Maternal smoking during pregnancy and smoking by adolescent daughters. Am J Public Health 84:1407-1413.

Kaufman J, Birmaher B, Brent D, Rao U, Flynn C, Moreci P, Williamson D, Ryan N (1997) Schedule for affective disorders and schizophrenia for 
school-age children-present and lifetime version (K-SADS-PL): initial reliability and validity data. J Am Acad Child Adolesc Psychiatry 36:980-988.

King SL, Marks MJ, Grady SR, Caldarone BJ, Koren AO, Mukhin AG, Collins AC, Picciotto MR (2003) Conditional expression in corticothalamic efferents reveals a developmental role for nicotinic acetylcholine receptors in modulation of passive avoidance behavior. J Neurosci 23:3837-3843.

Krmpotic-Nemanic J, Kostovic I, Kelovic Z, Nemanic D (1980) Development of acetylcholinesterase (AChE) staining in human fetal auditory cortex. Acta Otolaryngologica 89:388 -392.

Krmpotic-Nemanic J, Kostovic I, Kelovic Z, Nemanic D, Mrzljak L (1983a) Development of the human fetal auditory cortex: growth of afferent fibres. Acta Anat (Basel) 116:69-73.

Krmpotic-Nemanic J, Kostovic I, Nemanic D (1983b) The development of medial geniculate body in man: changes in the cholinesterase (CHE) activity during fetal and perinatal life. Acta Otolaryngologica 95:695-699.

Liang K, Poytress BS, Chen Y, Leslie FM, Weinberger NM, Metherate R (2006) Neonatal nicotine exposure impairs nicotine enhancement of central auditory processing and auditory learning in adult rats. Eur J Neurosci 24:857-866.

Liu Z, Neff RA, Berg DK (2006) Sequential interplay of nicotinic and GABAergic signaling guides neuronal development. Science 314:1610-1613.

Mabbott DJ, Noseworthy M, Bouffet E, Laughlin S, Rockel C (2006) White matter growth as a mechanism of cognitive development in children. NeuroImage 33:936-946.

McCartney JS, Fried PA, Watkinson B (1994) Central auditory processing in school-age children prenatally exposed to cigarette smoke. Neurotoxicol Teratol 16:269-276.

Meyers K, McLellan AT, Jaeger JL, Pettinati HM (1995) The development of the comprehensive addiction severity index for adolescents (CASI-A). An interview for assessing multiple problems of adolescents. J Subst Abuse Treat 12:181-193.

Mori S, Wakana S, Nagae-Poetscher LM, Van Zijl PCM (2005) MRI atlas of human white matter. Amsterdam: Elsevier.

Navarro HA, Seidler FJ, Eylers JP, Baker FE, Dobbins SS, Lappi SE, Slotkin TA (1989) Effects of prenatal nicotine exposure on development of central and peripheral cholinergic neurotransmitter systems. Evidence for cholinergic trophic influences in developing brain. J Pharmacol Exp Ther 251:894-900.

Oncken CA, Henry KM, Campbell WA, Kuhn CM, Slotkin TA, Kranzler HR (2003) Effect of maternal smoking on fetal catecholamine concentrations at birth. Pediatr Res 53:119-124.

Papademetris X, Jackowski AP, Schultz RT, Staib LH, Duncan JS (2003) Computing 3D non-rigid brain registrations using extended robust point matching for composite multisubject fMRI analysis. In: Medical image computing and computer assisted intervention (Ellis RE, Peters TM, eds), pp 788-795. Berlin: Springer.

Parent A (1996) Carpenter's human neuroanatomy. Baltimore: Williams and Wilkins.

Paus T, Zijdenbos A, Worsley K, Collins DL, Blumenthal J, Giedd JN, Rapoport JL, Evans AC (1999) Structural maturation of neural pathways in children and adoelscents: in vivo study. Science 283:1908-1911.

Payne BR (1992) Development of the auditory cortex. In: Development of auditory and vestibular systems 2 (Romand R, ed), pp 357-389. Amsterdam: Elsevier Science.

Payne BR, Pearson HE, Cornwell P (1988) Neocortical connections in fetal cats. Neurosci Res 5:513-543.
Perrot X, Ryvlin P, Isnard J, Guenot M, Catenoix H, Fischer C, Mauguiere F, Collet L (2006) Evidence for corticcofugal modulation of peripheral auditory activity in humans. Cereb Cortex 16:941-948.

Rogers SW, Gregori NZ, Carlson N, Gahring LC, Noble M (2001) Neuronal nicotininc acetylcholine receptor expression by $\mathrm{O} 2 \mathrm{~A} /$ oligodendrocyte progenitor cells. Glia 33:306-313.

Romano E, Tremblay RE, Farhat A, Cote S (2006) Development and prediction of hyperactive symptoms from 2 to 7 years in a population-based sample. Pediatrics 117:2101-2109.

Ruff RM, Parker SB (1993) Gender- and age-specific changes in motor speed and eye-hand coordination in adults: normative values for the Finger Tapping and Grooved Pegboard Tests. Percept Mot Skills 76:1219-1230.

Schmidt SL, Oliveira RM, Rocha FR, Abreu-Villaca Y (2000) Influences of handedness and gender on the Grooved Pegboard Test. Brain Cogn 44:445-454.

Shafritz KM, Marchione KE, Gore JC, Shaywitz SE, Shaywitz BA (2004) The effects of methylphenidate on neural systems of attention in attention deficit hyperactivity disorder. Am J Psychiatry 161:1990-1997.

Shaywitz BA, Shaywitz SE, Pugh KR, Fulbright RK, Skudlarski P, Mencl WE, Constable RT, Marchione KE, Fletcher JM, Klorman R, Lacadie C, Gore JC (2001) The functional neural architecture of components of attention in language-processing tasks. NeuroImage 13:601-612.

Shimony JS, McKinstry RC, Akbudak E, Aronovitz JA, Snyder AZ, Lori NF, Cull TS, Conturo TE (1999) Quantitative diffusion-tensor anisotropy brain MR imaging: normative human data and anatomic analysis. Radiology 212:770-784.

Slotkin TA (1998) Fetal nicotine or cocaine exposure: which one is worse? J Pharmacol Exp Ther 285:931-945.

Slotkin TA (2004) Cholinergic systems in brain development and disruption by neurotoxicants: nicotine, environmental tobacco smoke, organophosphates. Toxicol Appl Pharmacol 198:132-151.

Slotkin TA, MacKillop EA, Rudder CL, Ryde IT, Tate CA, Seidler FJ (2007) Permanent, sex-selective effects of prenatal or adolescent nicotine exposure, separately or sequentially, in rat brain regions: indices of cholinergic and serotonergic synaptic function, cell signaling, and neural cell number and size at 6 months of age. Neuropsychopharmacology 32:1082-1097.

Smith SM (2002) Fast robust automated brain extraction. Hum Brain Mapp 17:143-155.

Sobell LC, Sobell MB (1992) Timeline follow-back: a technique for assessing self-reported alcohol consumption. In: Measuring alcohol consumption: psychosocial and biological methods (Litten R, Allen J, eds), pp 41-72. Totowa, NJ: Humana.

Suga N, Xiao Z, Ma X, Ji W (2002) Plasticity and corticofugal modulation for hearing in adult animals. Neuron 36:9-18.

Sun X, Xia Q, Lai C, Shum DK, Chan Y, He J (2007) Corticofugal modulation of acoustically induced fos expression in the rat auditory pathway. J Comp Neurol 501:509-525.

Talairach J, Tournoux P (1988) Co-planar stereotaxic atlas of the human brain. New York: Thieme Medical.

Winer JA (2006) Decoding the auditory corticofgal systems. Hear Res 212:1-8.

Winer JA, Diehl JJ, Larue DT (2001) Projections of auditory cortex to the medial geniculate body of the cat. J Comp Neurol 430:27-55.

Woods RP (1996) Modeling for intergroup comparisons of imaging data. NeuroImage 4:S84-S94. 